\title{
Téoros
}

Revue de recherche en tourisme

\section{La formation dans le secteur de la restauration}

\section{Jeannine Cornellier et Ljubomir Téofilovic}

Volume 3, numéro 3, novembre 1984

Cuisine québécoise, restauration et tourisme : vers une synergie

féconde

URI : https://id.erudit.org/iderudit/1080780ar

DOI : https://doi.org/10.7202/1080780ar

Aller au sommaire du numéro

Éditeur(s)

Université du Québec à Montréal

ISSN

0712-8657 (imprimé)

1923-2705 (numérique)

Découvrir la revue

Citer cet article

Cornellier, J. \& Téofilovic, L. (1984). La formation dans le secteur de la restauration. Téoros, 3(3), 27-29. https://doi.org/10.7202/1080780ar d'utilisation que vous pouvez consulter en ligne.

https://apropos.erudit.org/fr/usagers/politique-dutilisation/ 


\title{
La formation dans le secteur de la restauration
}

\section{Les avantages de la formation scolaire}

\author{
par Jeannine Cornellier*
}

Des jeunes sont employés dans un restaurant. II s'agit là de leur première expérience de travail dans le milieu de la restauration. Ils n'ont recu aucune formation préalable relative à ce travail. Ils apprennent donc à sevir aux tables en même temps qu'ils font leur travail. Utilisant leurs ressources personnelles, ils observent les gestes des employés aseniorn, cherchent à maîtriser leur travail respectif, essaient d'acquérir des techniques qui leur sont plus ou moins bien

"Jeannine Cornellier est diététiste et professeure à l'Institut de touriame et d'hâtellerie du Quebec. explicitées et exécutent les tâches qui leur sont désignées. Ces personnes, n'ayant recu aucune formation dans une école spécialisé, se voient chargées d'accomplir une tâche auprès des clients. Dans un milieu oủ il faut agir vite et oủ la urentabilitén est I'objectif premier, chaque geste est integré lentement, très lentement. De plus, wl'ef= ficacitén s'avère le critère d'évaluation quotidienne des employés. Les jours passent et les jeunes travailleurs prennent conscience qu'une formation antérieure aurait facilité leur intégration dans le métier.

Essayons de comparer les comportements des jeunes qui arrivent bien préparés sur le marché du travail avec ceux des employés n'ayant reçu aucun entraine: ment. Les premiers ont bénéficié d'une formation structurée qui dose les apprentissages et les exercices, qui permet l'assimilation des connaissances, qui contrôle les acquisitions, qui corrige les défauts, qui permet les initiatives et qui laisse place à la virtuosité. Les employés non formés auraient peut-être désiré recevoir autre chose qu'une aformation sur le tasm. II est superflu de croire que le marché du travail poursuit des buts de formation. S'il s"agit d'une industrie de service à la clientèle, elle

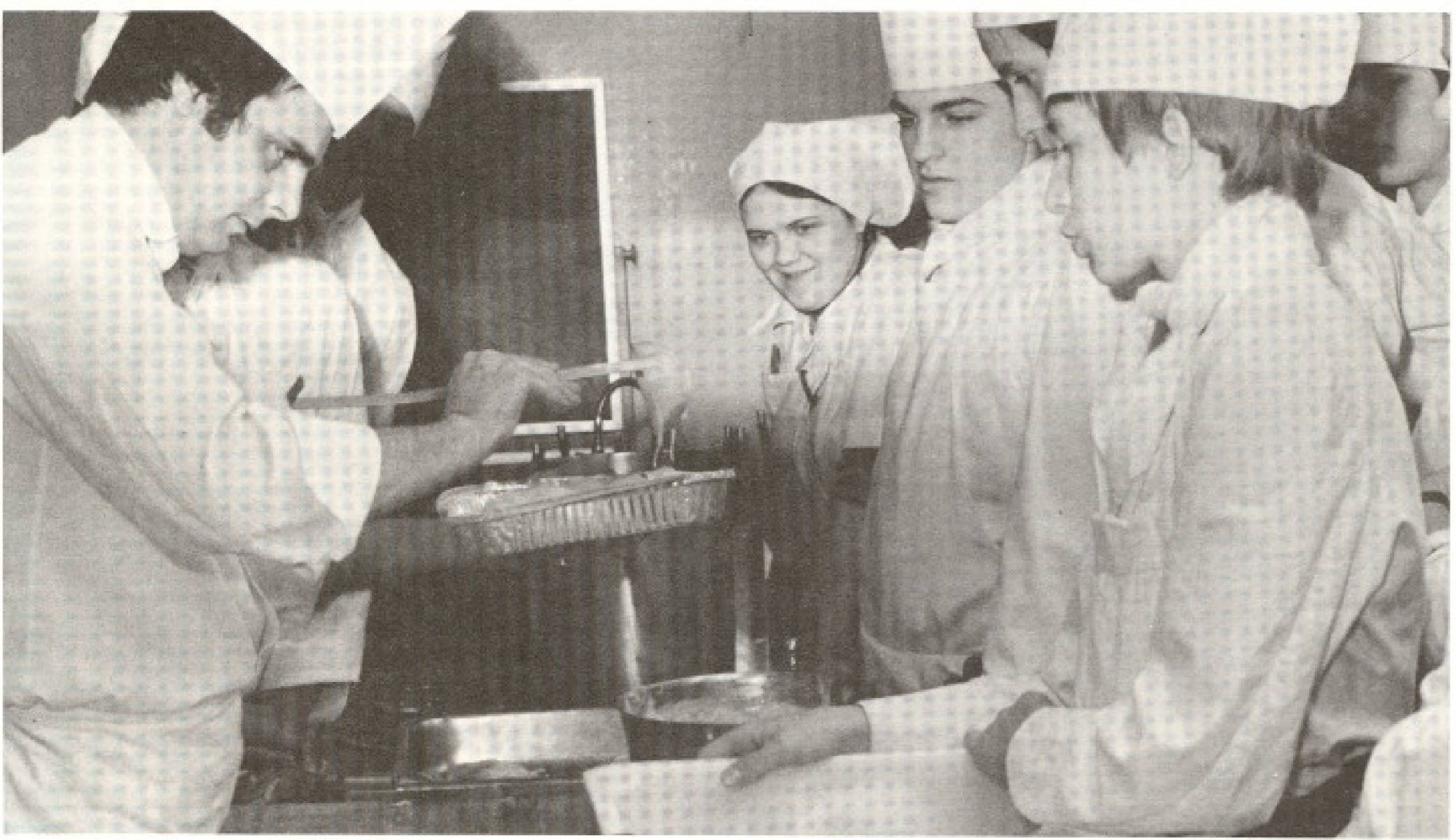

Une multitude de cours sont offerts à toute personne désireuse d'oeuvrer dans les domaines de la resitauration. 
cherche â rêpondre aux besoins de sa clientèle. Satisfaire une clientèle, certes! mais du même coup, il faut réaliser des profits. Méme si l'employeur affirme que le eprofit n'est pas une fin en soix, il faut bien comprendre que la compétence du personnel permet de réaliser non seulement des profits mais aide l'entreprise á parvenir au succès. Or, il s'avère difficile d'allouer du temps a la formation des employés et de diminuer d'autant le temps réservé à la qualité du service, â l'hygiène et a la propreté, personnel, à la promotion des ventes, à la qualité de l'administration et au dynamisme.

\section{Développer une capacité d'adaptation}

Le marché du travail poursuit des objectifs différents de ceux d'un programme d'enseignement. Celui-ci consiste, selon les différents grands pédagogues, aen un ensemble organisé de buts, d'objectifs spécifiques, de contenu organisé de facon séquentielle, de moyens didactiques, d'activites d'apprentissage et de procédés d'évaluation pour mesurer /'at" teinte de ces objectifsu. Larsque des jeu nes s'inscrivent à des programmes de formation professionnelle, ils s'attendent a recevoir un entrainement bien structuré afin de pouvoir atteindre un degré d'excellence dans l'exercice de la profession qu'ils ont choisie et ce, dans une période relativement courte, soit 2 ou 3 ans. Ces programmes d'études s'inscrivent alors au sein d'une conception de la formation professionnelle adoptée par certaines écoles de formation lex: Le Centre International de Glion, à travers le monde entier, à savoir: à la compétence et au comportement du

aproduire des individus capables de s'instruire et de s'adapter aux divers changements de situation qui interviennent tout au long de leur cheminement professionnel: (...) promouvoir de futurs responsables qui soient en mesure de passer du stade de l'idée a celvi de la réalisation pratique. avec tous les efforts de volonté et de controle qu'une telle démarche implique. (...) Ainsi, lorsque I'on parle de "former des futurs cadres (...), on entend ainsi par la non pas inculquer aux jeunes uniquement des connais: sances technologiques, mais aussiet surtout développer un esprit spécifique et entrainer les elèves à adopter des attitudes appropries dans des conditions proches de la réalité des entreprises..

Acquérir une formation professionnelle, vouloir se préparer ả exercer un métier, c'est développer une capacité d'adaptation sur le marché du travail, c'est contribuer à la promotion et au succès de mérite d'une formation visant l'excellence. Elle permet non seulement de bien connaître les techniques de base, de développer les attitudes et les comportements appropriés, mais aussi, elle rend apte à envisager toutes les situations nouvelles d'une façon traditionnelle. Ainsi, le savoir-être et le savoir-faire sont intégrés spontanément et s'épanouissent dans le succes.

Afin de répondre à ces besoins de savoir, de savoir-faire et de savoir-ettre, une multitude de cours sont offerts a toute personne désireuse d'oeuvrer dans les domaines de la restauration.

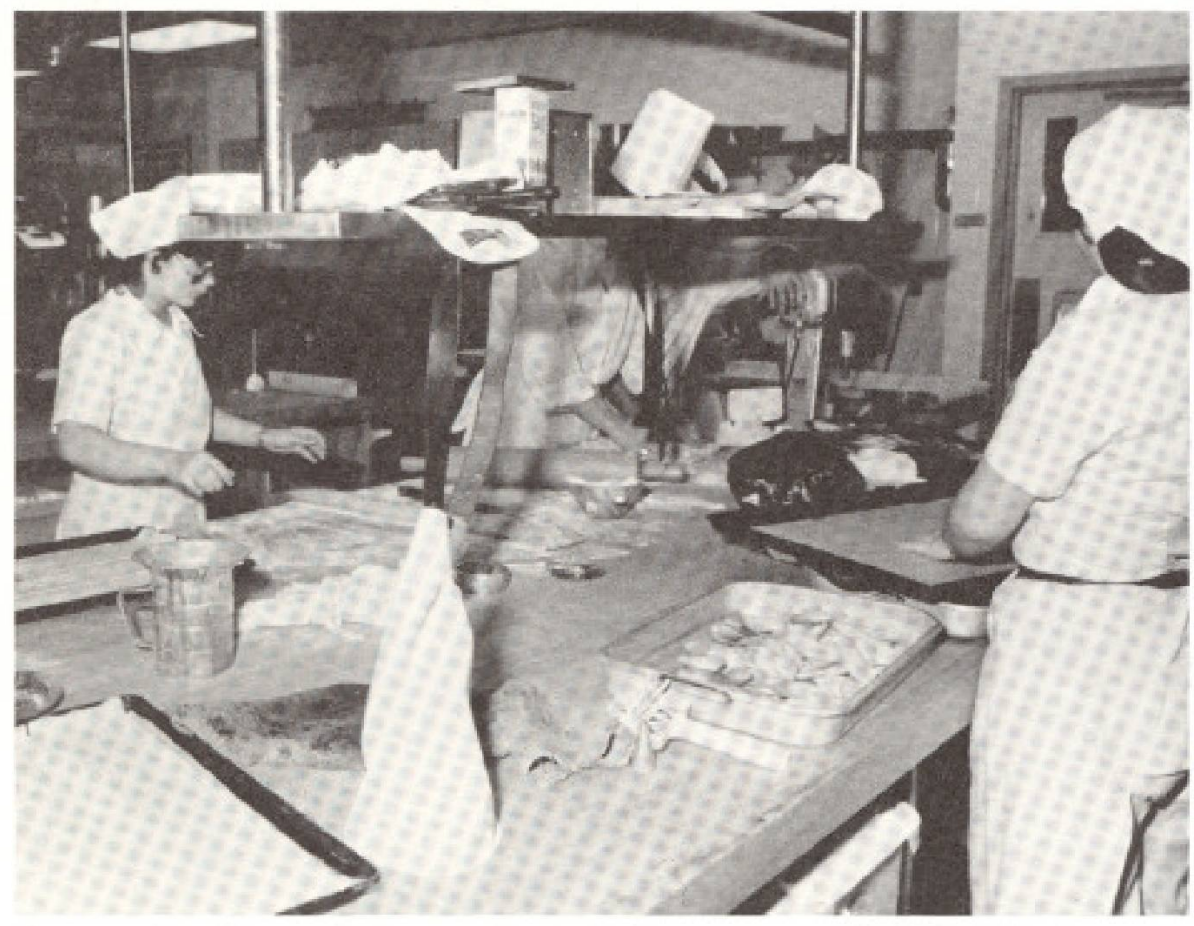

Des cours de cuisine professionnelle, de service de restaurant et de patisserie-boulangerie...
S'agit-il d'élèves réguliers de I'enseignement secondaire et postsecondaire, ils peuvent s'inscrire a des cours de cuisine professionnelle, de service de restaurant et de pátisserie-boulangerie. Ces cours sont dis: pensés par des organismes gouvernementaux ou privés ou par des commissions scolaires.

S'agit-il d'élèves réguliers du collégial, ils peuvent aussi s'inscrire à des programmes où, en plus d'étudier les principes de la gestion en restauration, ils reçoivent une formation en service de restaurant, en cuisine, en bar et en sommellerie.

S'agit-il enfin de personnes intéressées par l'éducation des adultes, il existe également pour elles une diversité de cours pouvant correspondre à la fois aux multiples besoins des secteurs de la restauration et ầ ceux des employés désireux d'y exercer un métier. Des cours de perfectionnement se donnent à temps partiel en cuisine professionnelle, gestion de cuisine, alimentation rationnelle, pătisserie-boulangerie, service de restaurant et service de bar. A ceux-ci peuvent s'ajouter des cours spécialisés, des cours sur mesure, des cours itinérants en service de bar et de restaurant, des sêminaires d'administration et des séminaires régionaux. De plus, des cours dits de recyclage sont dispensés â temps plein en cuisine d'établissement, gérance d'un centre hôtelier, service de bar et de restaurant et pâtisserie-boulangerie.

Les jeunes qui envisagent d'oeuvrer dans le secteur de la restauration peuvent être assurés de l'utilité d'une formation de qua: lité. Tenant compte des pratiques pédagogiques et disposant de contenus élaborés par des professionnels et des pédagogues. compétents, une telle formation constitue le moyen le plus rapide pour répondre aux besoins d'une industrie qui s'impose par son dynamisme et son pragmatisme, par son essor et ses traditions professionnelles, par son identité culturelle et ses racines. $f$

\section{Bibliographie}

CENTRE INTEANATIONAL DE GLION, Hōtelierie, Tourisme, Gestion hospitaliere, pochette publicitaire. janvier 1994

DEBEUR, T., Pierre Marcotte ou le respect du caph tal humain, L'Hospitalite, juillet 1984, pages 14 et 15 .

I.T.H.Q., Service de reducation des adultes, L'I.T.H,O. 15 ans apres, 1982, 24 pages.

NADEAU, M.-A., Evaluation des programmes d'étu des, Québec, Les Presses de l'Université Laval, 1981. 474 pages.

PLANCHARD, E., La recherche en pedagogie Paris, Beatrice-Nauwelaents Louvain, Editions Nauwelaents. 1967, 308 pages. 


\title{
Les programmes de formation font-ils place à la dimension touristique?
}

\author{
par Ljubomir Téofilovic*
}

Lorsque l'on s'occupe de formation, plus particulièrement de formation professionnelle, notamment dans le domaine du tourisme, il est difficile d'aborder un tel sujet sans se référer aux buts vișés par les différents programmes de formation conçus pour ce secteur de l'économie.

Voilă bien lả une attitude, un état d'esprit fortement marqué par ce que nous qualifierions de adéformation professionnelles puisque nous nous occupons précisément de formation.

Lè titre même de l'article nous suggère une double approche:

- cerner et définir en premier lieu, en quoi consiste la dimension touristique;

- vérifier ensuite si cette dimension est prise en considération et est contenue dans les programmes de formation relatifs au secteur de la restauration.

La dimension touristique dont il est question dans ce texte concerne exclusivement le client, soit-il touriste ou résident, qui se déplace, hors de son chez-soi, en quête d'un bon restaurant. Ce client a des besoins spécifiques et des exigences particulières qu'il importe de connailtre.

Dans l'éditorial de juillet-août 1984 de la revue Hôtellerie-Restauration, Gérard Delage écrit ceci: "N'oubliez jamais que I'auteur américain, dont je n'ai jamais su le nom, qui a eu la sagesse de decrire ('hotel lou le restaurant) comme wa home away from homew, ne voulait pas dire que nos établissements devaient ressembler a celles (sic) de nos clients. II voulait simplement laisser entendre qu'on devait y être aussi bien que chez soi. Autrement, pourquoi s'asseoir à une nouvelle table ou se coucher dans un nouveau it, sil'on mange mieux et sil'on dort plus agréablement dans sa propre maison? $n$.

Cette réflexion nous permet de relever un détail important. Celui qui se déplace désíre être partout aussi bien que chez lui. II s'agit là d'un besoin de sécurité que le client résident ou touriste cherche à combler. II accepte volontiers le dépaysement et se laisse aisement impressionner par des originalités en autant que cela n'ébranle pas ou ne modifie pas trop son confort quotidien, ses habitudes alimentaires et les coûts quiil débourse ordinairement pour ce genre de service.

* Ljubomir Téfilovic est coondonnateur de l'enseigne ment theorique a l'Institut de tourisme et d'hôtellerie du Quẻbec
Cette inquiétude que se crée le atéoross (celui qui voyage) se traduit par la recherche d'un accueil chaleureux et par une grande disponibilité et une compétence professionnelle de la part des préposés aux differents services auxquels il a recours. Sur le plan de la qualité de la table, de son originalité et de sa propreté, les exigences se révèlent tout aussi considérables. Bien entendu, ces critères d'exigence doivent cadrer avec les habitudes de vie du voyageur; autrement dit, ils équivalent ceux du foyer.

On pourrait rétorquer en soutenant que de chercher à satisfaire les besoins des clients s'avere une mission impossible puisqu'on compte autant de criteres qu'il $y$ a d'individus et de wchez-soin.

Tout en étant de cet avis, nous prétendons qu'il existe des normes générales qui font l'unanimité toute entière. Ainsi, nous voyons difficilement les clients s'insurger contre la propreté et l'hygiène des lieux ou le confort et la clarté découlant de l'aménagement des espaces. Personne ne se plaint jamais de l'amabilité des préposés aux services, de leur bonne disposition et de leur aide lorsqu'elle s'avère nécessaire. On n'a encore jamais vu un client refuser des mets qui lui ont êté bien proposés et dont la présentation est invitante et le goút correspondant à l'idée qu'ill s'était fait au départ. De plus, les clients consentent de bonne grăce à ce qu'on leur accorde une attention bienveillante. Enfin, rares sont les clients qui contestent une addition raisonnable lorsqu'ils ont été bien entourés et sécurisés.

Au sein de la restauration, toutes ces attitudes et ces comportements constituent, à notre avis, l'essence mëme de l'esprit touristique, de ce que nous appelions plus haut, la dimension touristique.

Ces préoccupations relevant du domaine des relations humaines se retrouvent-elles dans la composition des programmes de formation dans le secteur de la restauration?

A notre connaissance, il existe au Québec cinq programmes majeurs de formation professionnelle dans le secteur de la restauration. Trois d'entre eux visent à former des travailleurs spécialisés en cuisine pro. fessionnelle, en pâtisserie-boulangerie et en service de restaurant. Les deux autres ont pour objectif de former des cadres intermédiaires qualifiés en gestion des services alimentaires et en gestion hótelière.
Aprés la lecture des objectifs de chacun de ces programmes, nous demeurons quelque peu surpris de constater que seules les connaissances et les attitudes techniques sont mentionnées.

En effet, on apprend que le programme de cuisine professionnelle a pour but de former des candidats aptes à occuper des postes de cuisiniers (ières) dans les différents établissements. Le programme en service de restaurant a pour but de spécialiser l'élève en lui enseignant toutes les techniques du métier afin qu'il puisse développer les habiletés et les comportements d'un(e) serveur(euse) accompli(e). Les programmes de gestion visent a foumir aux futurs gestionnaires une formation complète en cuisine et en service de restaurant tout en leur donnant une solide formation en gestion (prix de revient, vente, approvisionnement, inventaire, personnel, etc.)

Mais il reste à sasvoir si ces profils de formation professionnelle sont réellement aussi techniques et aussi dépourvus des aspects humains tant recherchés par le client.

La technique en elle-même ne consiste-telle pas à exécuter une oeuvre ou un acte dans la règle de l'art exigée par cette mềme oeuvre ou ce même acte? En conséquence, puisqu'il s'agit d'apprentissage de techniques d'accueil au sens large du mot, les techniques enseignées contiennent implicitement les comportements humains et les attitudes professionnelles mentionnées plus haut. La subtilité réside dans la vigilance à faire acquérir aux apprentis la totalité des attitudes propres à chaque technique, ce qui correspond aux désirs du client.

La dimension touristique en matięre de formation dans le secteur de la restauration se révèle donc omniprésente et implicite dans les différents programmes de formation professionnelle pour l'industrie du tourisme. Cependant, il reste à développer une vigilance aiguë et constante dans l'enseignement des différentes techniques professionnelles d'accueil pour s'assurer que les moindres détails, qui font toute la différence dans la qualité, sont non seulement transmis, mais également et surtout professionnellement acquis par l'élève. f 